\title{
Comparison of Determined Stellar Parameters between LAMOST and SEGUE Spectra
}

\author{
Yue $\mathbf{W u}{ }^{1,2}$ Ali Luo ${ }^{1,2}$ Bing $\mathbf{D u}^{1,2}$ and Yanxin Guo ${ }^{1,2}$ \\ ${ }^{1}$ National Astronomical Observatories, Chinese Academy of Sciences, \\ 20A Datun Road, 100012, Beijing, China (NAOC) email: wuyue@lamost.org \\ ${ }^{2}$ Key Laboratory of Optical Astronomy, NAOC, Chinese Academy of Sciences, China
}

\begin{abstract}
We positionally cross-matched the stellar observations between LAMOST pilot survey and SDSS/SEGUE database, picked out more than 4000 targets observed by both projects, mostly are late A and FGK type stars. For the two dataset, we adopted ULySS program (Koleva et al. 2009, Wu et al. 2011a) to determine the stellar atmospheric parameters $\left(T_{\text {eff }}\right.$, $\log g,[\mathrm{Fe} / \mathrm{H}]$, Radial Velocity) with the ELODIE library as a reference (Wu et al. 2011b). For the individual parameter, we made two kinds of comparison, first for the SDSS spectra, second for the LAMOST spectra, tested the differences between the SSPP (SDSS/SEGUE stellar parameter pipeline) measurements and the ULySS derived results. Since the LAMOST pilot survey observations SNR (Signal to Noise Ratio) are low, for the latter comparison we excluded those stars with $g$ band SNR $<10$, then the sample volumn reduced to around 1300. Fig.1 displays the details of the second kind comparison for each parameter. All the comparisons demonstrate acceptable and reasonable agreement between each other for medium-resolution survey spectra. Our preliminary examination on the precision of the stellar parameters that could be derived from the LAMOST pilot survey observations exhibits the equivalent capability of LAMOST to study our Milky Way as for the SDSS/SEGUE. The LAMOST telescope executed its formal survey from Sep. $28^{\text {th }} 2012$, it will yield more better quality stellar spectroscopic data spanning in deeper and broader sky coverage (this work was supported by the Natural Science Foundation of China under No. 11103031).
\end{abstract}

Keywords. stars: fundamental parameters - techniques: spectroscopic - galaxies: stellar content
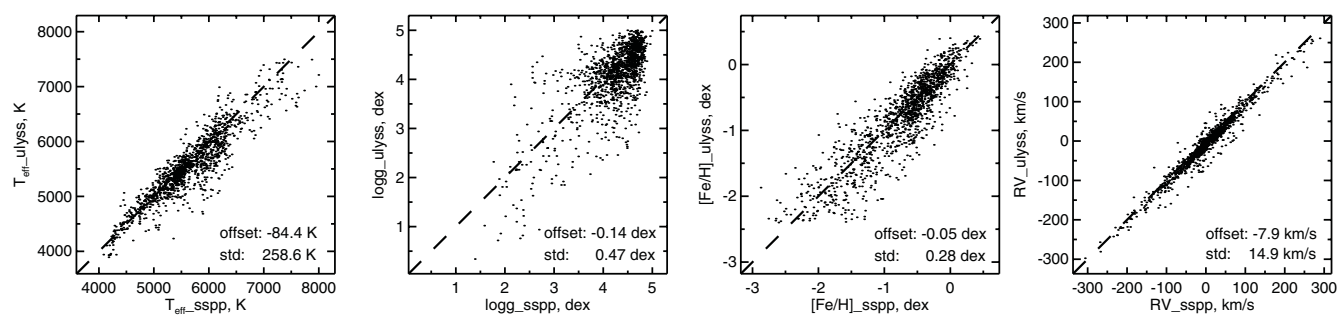

Figure 1. Comparisons of the parameters. The abscissa shows the SSPP measurements, while the ordinate indicates the ULySS ones (LAMOST spectra). The offset and $1 \sigma$ standard deviation between the comparing two series are listed on each panel. The dashed lines are the 1:1 ratios.

\section{References}

Koleva, M., Prugniel, P., Bouchard, A., \& Wu, Y. 2009, A\&A, 501, 1269

Wu, Y., Luo, Ali, Li, H. N., et al. 2011a, RAA, 11, 924

Wu, Y., Singh, H. P., Prugniel, P., et al. 2011b, A\&A, 525, 71 